\title{
Lane Detection for Intelligent Vehicle under Different Illumination Conditions
}

\author{
Pingshu $\mathrm{Ge}^{1}$, Lie Guo ${ }^{2,}$, Tao Zhang ${ }^{1,3}$, Xiuchun Zhao ${ }^{1,3}$ and Jing Chang ${ }^{2}$ \\ ${ }^{1}$ College of Mechanical and Electronic Engineering, Dalian Minzu University, Dalian, China, 116600 \\ ${ }^{2}$ School of Automotive Engineering, Dalian University of Technology, Dalian, China, 116024 \\ ${ }^{3}$ Faculty of Electronic Information and Electrical Engineering, Dalian University of Technology, Dalian, China, 116024 \\ * Corresponding author
}

\begin{abstract}
Lane detection is an important component for intelligent vehicle. A novel method for lane detection under complex illumination conditions is presented. An adaptive image segmentation technology based on OTSU algorithm was used to segment lane markings by combining the global and the local threshold. The improved Sobel operator was adapted to abstract the edges of the lane markings. The lane was finally identified using the improved Hough transform by limiting the range of lane parameters. On road experiments show that the proposed method has good accuracy and real-time performance under different illumination conditions.
\end{abstract}

Keywords-intelligent vehicle; lane detection; linear lane model; Hough transform

\section{INTRODUCTION}

Safety is a hot topic for automotive industries and has gained much concern recently. Developing intelligent vehicle is one of the effective methods to reduce traffic accidents, whose main objective is to increase driver awareness by providing useful information [1].

One of the most frequent causes of highway accidents is the unintended lane deviation, because of inattention, sleepiness or drowsiness. To avoid such kind of traffic accidents, lane departure warning system (LDWS) is researched worldwide aiming to effectively reduce or avoid the traffic accident. Accurate to detect the lane is an important component of LDWS [2]. The typical vision based lane detection methods can be classified into feature based and model based. Feature based method mainly utilizes some characteristic of the road image, such as gray gradient features, to identify the lane. This method is suitable to most kinds of road shape. When the road image is complex, the algorithm may need much time to complete the edge detection and will reduce the real-time performance of the system. Model based method uses several geometrical elements to describe the lanes, such as parabolic curves, hyperbola and straight lines [3]. This method mainly oriented to structured road with the corresponding road model according to the lane line shape. For example, Yoo et al. [4] presented a piecewise linear model based on Hough transform. Model based method is able to ensure good recognition accuracy, but the recognition effect is poor under complex illumination conditions.

This paper presents a new lane detection algorithm for different conditions. The image processing is limited in the lane region of interest. An adaptive image segmentation method is used to realize the segmentation of lane markings. According to the linear lane model, an improved Hough transform is adopted to identify the lane. Experiments are conducted to verify the proposed lane detection method.

\section{THE LINEAR LANE MODEL}

Model based lane detection method can overcome the influence of shadow and lane boundaries damage. How to choose an appropriate lane model is important for the lane detection algorithm [5]. The generally used lane model are curve model and piecewise linear model, which are time consuming and demand complex computations. Instead, this paper utilizes the linear lane model. The error of the linear lane model used in the paper is illustrated in Figure 1.

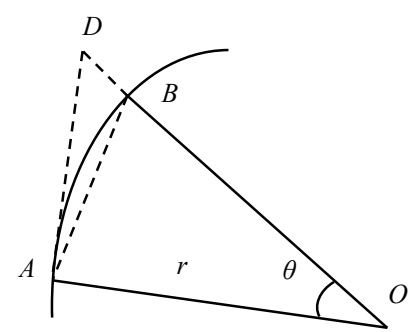

FIGURE I. ERROR DIAGRAM OF THE LINEAR LANE MODEL

According to the technical standard of highway engineering and related documents, the minimum curve radius of standard expressway is $650 \mathrm{~m}$. The lane marking, which is approximately $60 \mathrm{~m}$ ahead of the vehicle, can be approximated as a straight line. Parameters can be defined as follows. The curve radius $r$ is $650 \mathrm{~m}$. In the image, the lane marking in front of the vehicle is a circular arc, the length of which is $\square_{A} B=60 \mathrm{~m}$. The corresponding central angle is $\theta$. Straight line $\mathrm{AD}$ is tangent to the arc $4 \mathrm{~A} B$. Point $\mathrm{A}$ is the point of tangency and point $\mathrm{D}$ is the intersection of the extension of line $\mathrm{AD}$ and OB. According to the relationship between the angle and perimeter, that is

$$
\frac{60}{2 \pi r}=\frac{\theta}{360^{\circ}}
$$

Then the central angle can be calculated. 


$$
\theta=\frac{60 \times 360}{2 \pi r} \approx 5.29^{\circ}
$$

The length of the straight line can be achieved as

$$
\left\{\begin{array}{l}
|A D|=r \tan \theta=650 \times \tan 5.29^{\circ}=60.15(\mathrm{~m}) \\
|A B|=2 r \sin \frac{\theta}{2}=2 \times 650 \times \sin \frac{5.29^{\circ}}{2}=59.96(\mathrm{~m})
\end{array}\right.
$$

If the real line marking is represented by the straight line $\mathrm{AD}$ or $\mathrm{AB}$, the corresponding deviation is $0.25 \%$ and $0.07 \%$, respectively. It indicates that the length deviations are less than $0.25 \%$, which is a small value. Therefore, the real lane marking can be substituted by a straight line. To enhance the real-time performance of the lane detection algorithm, the relatively simple linear lane model is utilized.

\section{LANE DETECTION}

The camera usually mounted on the front windshield or the rear view mirror of a vehicle with a certain pitch angle. The image acquired by the camera is constituted by the sky area and the road area. By regulating the pitch angle of the camera, the sky area can be adjusted to locate in the top half of the image. Whereas, the bottom half of the image can be selected as the region of interest (ROI), which helps to improve the calculation speed.

\section{A. Image Filtering}

The improved median filtering method is adopted to reduce noise interferences. A $3 \times 3$ filtering window is utilized to progressively scan the ROI. Each pixel is evaluated by judging whether it is the extremum value covered by the filtering window. The pixel is retained to be processed by the normal median filter if the pixel has an extreme value. Otherwise, it will not be processed.

To verify the effect of image filtering algorithm, the salt and pepper noise with a frequency of 0.02 is added to the road image. Then, the road image with noise is processed by the above filtering method. The filtering results are shown in Figure 2.

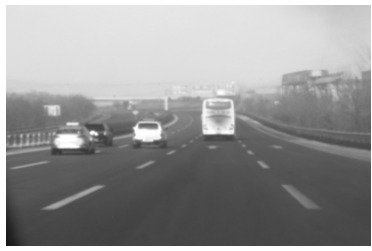

(a) The original road image
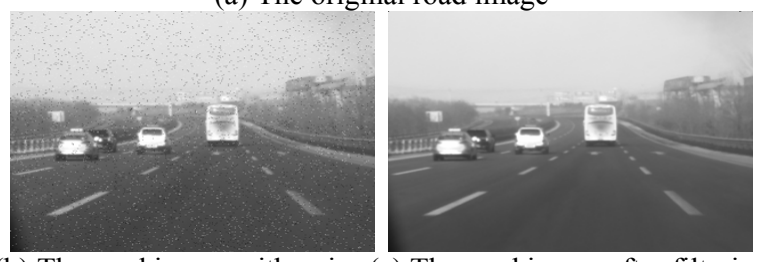

(b) The road image with noise (c) The road image after filtering FIGURE II. RESULT OF IMAGE FILTERING ALGORITHM
Results indicate that this method can eliminate the burst noise without effect on the edge abstraction.

\section{B. Adaptive Image Segmentation}

The selection of threshold value is the key issue to fulfill the image segmentation, although it is a difficult issue for most image processing applications. The traditional OTSU algorithm shows better segmentation results for normal illumination conditions [6]. Whereas, when the road images are low contrast images, the effect is not ideal. Therefore, this paper proposes an adaptive image segmentation method based on OTSU algorithm by combining the global threshold determined by OTSU algorithm and the local threshold determined by the local threshold method to segment the road image.

The global threshold $T_{0}$ is calculated utilizing the traditional OTSU algorithm firstly. A $3 \times 3$ neighborhood window slides through the image to determine the local threshold $T_{\text {mean }}$ for each pixel. Then the global and local thresholds are weighted to get the adaptive threshold $T$ for the current pixel, that is

$$
T=T_{0} \times(1-\omega)+T_{\text {mean }} \times \omega
$$

where $\omega$ is the weight, which is an empirical value determined according to experiment results.

Figure 3 compares the segmentation results of the traditional OTSU method and the proposed adaptive method under different illumination conditions. The left column images are the results utilizing the traditional OTSU method, while the right column images show the results of the proposed method under corresponding conditions. Results indicate the proposed method can effectively avoid the influence of different light conditions on lane markings.
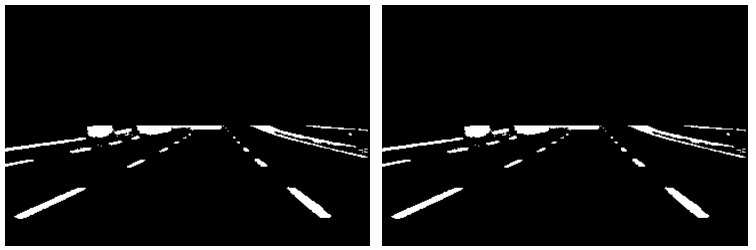

(a) Normal light condition

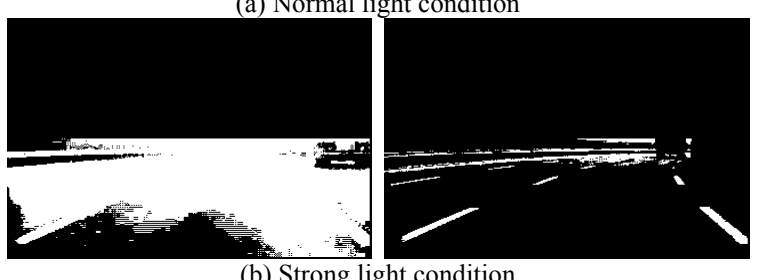

(b) Strong light condition

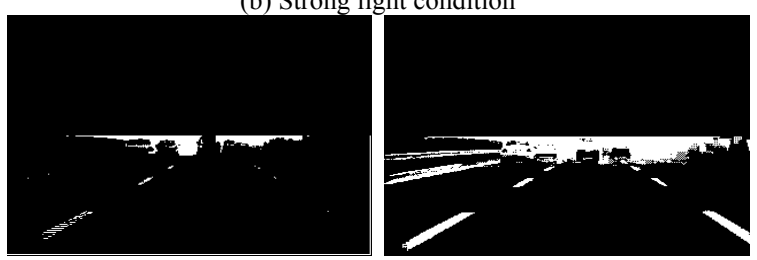

(c) Weak light condition 


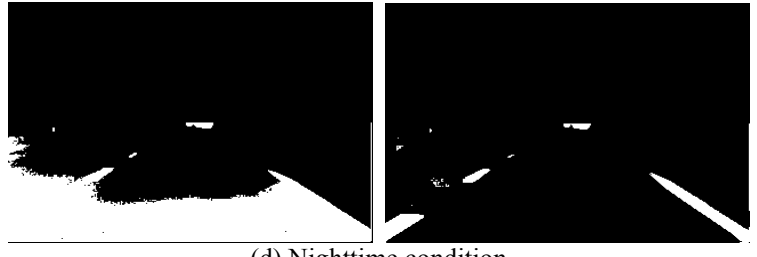

(d) Nighttime condition

FIGURE III. COMPARISON OF IMAGE SEGMENTATION

\section{Edge Detection}

Edge is important information for lane detection. Edge detection is to find the mutation points of the gray value in the image. Among common edge operators, Sobel operator shows good performance to eliminate most of the useless information and noise [7]. This paper adopts the Sobel operator to abstract the edges of the lane markings. After image filtering, most of the lane markings in the image are reserved. To better enhance the edges of the left and right lane markings, the Sobel operator templates of $45^{\circ}$ and $135^{\circ}$ directions, as shown in Figure 4, are utilized to process the road images after image segmentation.

\begin{tabular}{|c|c|c|}
\hline-2 & -1 & 0 \\
\hline-1 & 0 & 1 \\
\hline 0 & 1 & 2 \\
\hline
\end{tabular}

\begin{tabular}{|c|c|c|}
\hline 0 & 1 & 2 \\
\hline-1 & 0 & 1 \\
\hline-2 & -1 & 0 \\
\hline
\end{tabular}

(a) $45^{\circ}$ gradient direction

(b) $135^{\circ}$ gradient direction

FIGURE IV. IMPROVED SOBEL OPERATOR MODELS

\section{Lane Detection}

Lane detection is the basis for LDWS to realize its warning function. The Hough transform method is the commonly used method for lane detection. Hough transform is able to detect the linear targets in the image space by mapping to the parameter space. The traditional Hough transform can be used to detect all the likely straight lines in the image by processing on the edge detection image. Whereas, the traditional Hough transform has some disadvantages when used for detecting the lane lines. First of all, all the pixels are to be processed in the statistic of the parameter space, which result in the increasement of the calculated amount and time consuming. Furthermore, the existence of a straight lane line in image is to judge whether the local maximum exceeds a certain threshold or not [8]. If the maximum of the accumulator is produced by the false peak distribution effect, it will result in misidentification.

Based on the above disadvantages, an improved Hough transform method is proposed. It can avoid the misidentification of lane line which produced by the false peak distribution, as well as to reduce the running time of the algorithm. The detailed steps are as follows.

(1) The straight line in the image space can be represented in the following parametric format using the Hesse normal form

$$
\rho=y \cos \theta+x \sin \theta
$$

where $\rho$ is the distance from the coordinate origin to the closest point of the straight line, and $\theta$ is the angle between the $y$ axis and the straight line.

The $x-y$ Cartesian coordinate system of the image is shown in Figure 5, where the origin is the lowest center point of the road image. The image is divided into two parts. Commonly, most parts of the left lane line will fall in the left half of the image, while most parts of the right lane line will fall in the right half of the image.

2) To reduce time consuming, the parameters $\rho$ and $\theta$ are quantized and limited according to the ranges of the possible left and right lane position in the image [9]. After analyzing the road experiments results, the angles between the left and right lane and the $y$ axis, symbolled by $\theta_{l}$ and $\theta_{r}$ respectively, are mostly in the range of $\left[25^{\circ}, 65^{\circ}\right]$. While the distances from the origin to the left and right lane, symbolled by $\rho_{l}$ and $\rho_{r}$ respectively, are in the range of $\left[0, \rho_{\max }\right]$. The maximum distance $\rho_{\max }$ can be calculated as

$$
\rho_{\max }=\sqrt{H^{2}+\left(\frac{W}{2}\right)^{2}}
$$

where $H$ and $W$ is the image height and width respectively.

3) Quantify the parameter space $(\rho, \theta)$ and build a two dimensional accumulator for the left and right image respectively. One dimension of the accumulator is the quantized angle $\theta$ and the other dimension is the quantized distance $\rho$. The step for the angle is $0.5^{\circ}$. For each edge point at $(x, y)$, the Hough transform algorithm determines if there is enough evidence of a straight line at that point. If so, it will accumulate the pair $(\rho, \theta)$ of that line, and then look for the accumulator's bin that the parameters may fall in. The value of the bin will increase correspondingly. Therefore, each element of the accumulator has a value equal to the sum of the edge points that may position on the line represented by the pair $(\rho$, $\theta$ ). By looking for the local maxima of the accumulator, the parameters for the left and right lane lines can be extracted and converted to parameters of lane lines in the Cartesian coordinate system.

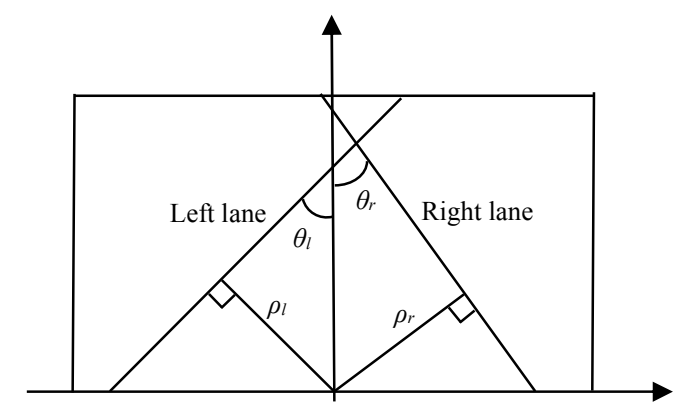

FIGURE V. CARTESIAN COORDINATE SYSTEM OF IMAGE 


\section{EXPERIMENT AND DISCUSSION}

To verify the effectiveness and efficiency of the proposed lane detection method, several experiments were conducted on real highway scenarios. The driving environment is focused on highway with different illumination conditions. The experiment system is programmed using VS2005. The size of the road image is $320 \times 240$ pixels captured by a vehicle mounted camera. The experiments were carried on the following specific illumination conditions: normal light, strong light, weak light, and nighttime condition. Some of the experimental results are shown in Figure 6.

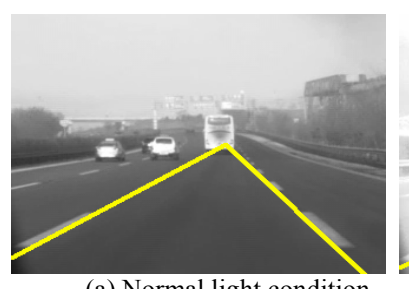

(a) Normal light condition

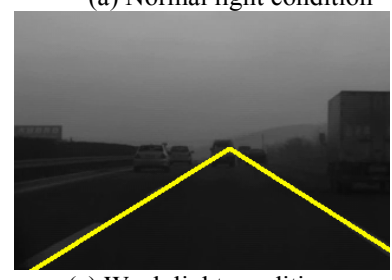

(c) Weak light condition

FIGURE VI. LANE DETECTION RESULTS ON REAL ROAD CONDITIONS

Moreover, to better present the performance of the proposed method, several comparisons are conducted under different illumination conditions with the traditional Hough transform based lane detection method. A total of 200 frame images are selected for each conditions to conduct the comparisons. The results are shown in Table 1.

\section{TABLE 1 COMPARISON OF EXPERIMENTAL RESULTS}

\begin{tabular}{|c|c|c|c|c|c|}
\hline Method & Condition & $\begin{array}{c}\text { Number of } \\
\text { image }\end{array}$ & $\begin{array}{c}\text { Number of } \\
\text { correct detec } \\
\text { image }\end{array}$ & $\begin{array}{c}\text { Accuracy } \\
(\%)\end{array}$ & $\begin{array}{c}\text { Average } \\
\text { time } \\
(\mathrm{ms})\end{array}$ \\
\hline \multirow{2}{*}{$\begin{array}{c}\text { Traditional } \\
\text { Hough } \\
\text { transform } \\
\text { method }\end{array}$} & normal & 200 & 182 & 91 & 89.35 \\
\cline { 2 - 6 } & strong & 200 & 175 & 87.5 & 90.17 \\
\cline { 2 - 6 } & neak & 200 & 170 & 85 & 91.76 \\
\hline \multirow{2}{*}{$\begin{array}{c}\text { The } \\
\text { proposed } \\
\text { method }\end{array}$} & normal & 200 & 173 & 86.5 & 93.54 \\
\cline { 2 - 6 } & strong & 200 & 188 & 96 & 56.87 \\
\cline { 2 - 6 } & weak & 200 & 185 & 92.5 & 56.93 \\
\hline \multirow{2}{*}{ nighttime } & 200 & 183 & 91.5 & 58.25 \\
\hline
\end{tabular}

Table 1 shows that the proposed lane detection method can realize a recognition accuracy of $91.5 \%$ in nighttime condition, which is improved by $5.7 \%$ compared with the traditional Hough transform. Moreover, the average processing time is decreased for all the illumination conditions. The results show that the proposed lane detection method has better anti-disturbance performance and can adapt to different illumination conditions with a higher recognition accuracy. The average processing time for each image is $57.35 \mathrm{~ms}$, which is 17 frames per second, which means that the algorithm can meet the real-time requirements for automotive applications.

\section{V.CONCLUSION}

The traditional lane detection methods fail to adapt to different illumination conditions, this paper proposes a new lane detection method by enhancing the anti-disturbance ability and recognition accuracy.

1) To segment the image and retain most useful information for lane detection, an adaptive segmentation algorithm based on OTSU is proposed, which combines the global and the local threshold to segment the image.

2) For the time-consuming problem of the traditional Hough, an improved Hough transform method is presented to search the left and right lane areas and limit the range of lane parameters, which can effectively reduce the computation complexity and improve the real-time performance.

3) This proposed method can realize a higher accuracy under different illumination conditions and the algorithm can meet the requirements for automotive applications.

\section{ACKNOWLEDGEMENT}

This work was supported by the National Natural Science Foundation of China under Grant Number (51575079) and the Fundamental Research Funds for the Central Universities under Grant Numbers (DUT16QY10, DCPY2017010 and DC201502010306).

\section{REFERENCES}

[1] D. Martín, F. García, B. Musleh, D. Olmeda, G. Peláez, P. Marín, A. Ponz, C. Rodríguez, A. Al-Kaff, A. De La Escalera, J. M. Armingol, "IVVI 2.0: An intelligent vehicle based on computational perception," Expert Systems with Applications, vol.41, no.17, pp.7927-7944, 2014.

[2] J. Son, H. Yoo, S. Kim, and K. Sohn, "Real-time illumination invariant lane detection for lane departure warning system," Expert Systems with Applications, vol.42, no.4, pp.1816-1824, 2015.

[3] Đ. Obradović, Z. Konjović, E. Pap, and I.J. Rudas, "Linear fuzzy space based road lane model and detection," Knowledge-Based Systems, vol.38, pp.37-47, 2013

[4] H. Yoo, U. Yang, and K. Sohn, "Gradient-enhancing conversion for illumination-robust lane detection," IEEE Trans. Intell. Transp. Syst., vol.14, no.3, pp.1083-1094, 2013.

[5] J. Ruyi, K. Reinhard, V. Tobi, and W.Shigang, "Lane detection and tracking using a new lane model and distance transform," Machine Vision and Applications, vol.22, no.4, pp.721-737, 2011.

[6] C.D. Cruz, and Z. Ju Jia, editors. , "Lane detection for driver assistance and intelligent vehicle applications," International Symposium on Communications and Information Technologies, pp.17-19, Oct. 2007.

[7] L. Qing, Z. Nanning, and C. Hong, "Springrobot: a prototype autonomous vehicle and its algorithms for lane detection," IEEE Trans. Intell. Transp. Syst., vol.5, no.4, pp.300-308, 2004.

[8] C.G. Ho, R.C.D. Young, C.D. Bradfield, and C.R. Chatwin, "A fast hough transform for the parametrisation of straight lines using fourier methods," Real-Time Imaging, vol.6, no.2, pp.113-127, 2000.

[9] P.S. Ge, L. Guo, G.K. Xu, R.H. Zhang, and T. Zhang, "A real-time lane detection algorithm based on intelligent CCD parameters regulation," Discrete Dynamics in Nature and Society, 2012, Article ID 273164, 16 pages, doi:10.1155/2012/273164. 